\title{
ORDEN CURRICULAR Y ESTRUCTURAS DE CONOCIMIENTO
}

\author{
JAIME CASTAÑÉ CASELLAS \\ Universidad Complutense de Madrid
}

Conocer de modo ordenado y adquirir conocimiento progresivo gracias al orden, son aspectos de una solución que el hombre requiere desde el propio nucleo humano, definido por su necesidad y poder de construirse: se construye guiado por esa solución sobre la base y con recursos ya adscritos al modo humano de ser, a la vez condicionado y capaz de llevar la pregunta hasta la articulación dinámica de sus condiciones y aun hasta la raíz de ellas, y así en algunos aspectos dominarlas.

Encontrar dicho orden del conocimiento y de su adquisición progresiva desde el punto de vista pedagógico significa hallar coherencia, como vinculación recíproca a través de la cual se constituyen y desarrollan los conocimientos de modo unitario; pero con un carácter específico significa además llevar esta coherencia cognoscitiva al proceso de construirse el hombre desde sí mismo según los valores, o sea, según lo que en relación con las propias exigencias humanas de ser más, se le muestra merecedor de estima por ofrecerle el cómo y también el porqué de tal realización. En el plano del conocimiento, debe la pedagogía articular el proceso y hacer efectiva una racionalidad que respecto de él logre traducirse en intervención capaz de darle curso según la relación entre los valores y quienes se educan al asumirlos. Ahora bien, así concebida la racionalidad pedagógica, ha de cumplirse en el ajuste concreto de su coherencia a las condiciones y de manera más radical a las necesidades que la realidad educativa le impone; y por otra parte, debe dar respuesta a la doble dimensión del orden interhumano y del que es preciso desarrollar en cada uno de los distintos sujetos o individuos educables.

Importa sobremanera preguntase por la interdependencia y la armonía de las dos dimensiones citadas, refiriendo la interrogación a las condiciones y necesidades impuesta a la racionalidad pedagógica; y en definitiva, interrogando sobre la tarea de construirse el hombre desde sí mismo, gracias a la intervención educativa como uno de los recursos dinámicos que deben incidir en él para que su vida sea proceso de construcción humana según los valores. Aquí la pregunta se explicita por su referencia al orden curricular, 
y en él a caracteres y condiciones de índole interhumana común que lo determinan, y por su referencia más radical a las estructuras de conocimiento que deben formarse en los individuos humanos a través del quebacer educativo, mediador entre la cultura y las experiencias individuales.

Orden humano: su construcción por el conocimiento

Las informaciones sistematizadas en el sujeto de educación al que se refiere la intervención educativa, han de traducirse en modificación desde el mismo sujeto, que recibiéndolas según cierto orden, a su vez las reorganiza, y ordena la propia manera humana de comportarse y de ser según posibilidades que este conocimiento le descubre. La pregunta por el orden curricular obliga a prestar atención a "los conocimientos y modelos comportamentales que puede transmitir la comunicación educativa". (Sarramona, 1983, p. 55). Se trata de lograr así desde dicho orden la realización de un proceso que por su carácter educativo "consiste en la regulación del comportamiento humano en virtud de la referencia a un estado final adoptado como principio de valor". (Vázquez, 1985, p. 177). En relación con la citada pregunta por el orden curricular, es claro que la respuesta no viene dada por la sola y simple "estructura de fines". Después de considerar Westbury lo incompleto de tal concepción, explica su propio concepto por analogía con la relación íntima que en el culto religioso guardan la Liturgia y la Teología, en las cuales, en su opinión, la segunda lleva necesariamente consigo determinadas formas de la primera, y ésta presupone también a la Teología para tener sentido y poder desarrollarse: no menos indisociable y fuerte es la unidad establecida, según el orden o coherencia curricular, entre la teoría sobre los fines y medios y los elementos materiales que cobran configuración y cumplen función didácticas y educativas.

Así, diferentes modos de entender "la naturaleza y los medios propios de la educación, tienen implicaciones diferentes que se traducen en las formas del aula". (Westbury, 1980, p. 100). Como Scwab advierte, "mal puede el curriculum relacionarse con sus realidades efectivas, si las trata solamente como copias de sus representaciones teóricas" (1971, p. 319). Hay, sin duda, razón para definir el orden curricular por la unidad entre la teoría -sobre fines y medios de educación-y las formas concretas adoptadas por los elementos de la teoría al hacerse efectivos en relación con ella: "Términos tales como actividad, estructuras, conocimietno y otros parecidos son abstracciones. Ocupan el lugar de cosas que en el mundo cotidiano de las escuelas son los temas, períodos, aulas y lecciones". (Westbury, 1980, p. 90). ¿Cómo relacionar adecuadamente el orden del sistema así construido y el que en el educando se debe construir, a través de la intervención sobre las estructuras individuales de conocimiento? 
La interconexión de elementos que constituye el sistema, y el orden entre sus relaciones constitutivas que le da carácter de estructura, aquí aparecen como interdependencia curricular, establecida a través de fines y medios, en el plano teórico y a nivel de efectividad concreta, por quienes asumen tal sistema y la correspondiente estructura. Ahora bien, entre las implicaciones del orden curricular que de este modo se construye, es la más significativa su referencia al quehacer educativo como tarea de construir al hombre. Se trata de interdependencia curricular definida por la de mensajes optimizadores que incidan en el educando, le muestren posibilidades de realización según los valores educativos, y al mostrárselas le permitan organizar experien. cias -sistemetizarlas como síntesis de información- por las cuales logre dar estructura a los propios cauces de conocimiento, y al conocer de esta forma, consiga realizarse como hombre desde sí mismo, supuestas ciertas condiciones entre las que se encuentra la mediación curricular. En la doble perspectiva de influjos mediadores y despliegue individual suscitado por el sistema que los produce, "la educación se nos presenta como el instrumento de construcción humana más potente, que al ser generado por el propio hombre debe estar 'más' sometido a sus aspiraciones, deseos y utopías". (Castillejo, 1986, p. 25).

Al establecer así el sistema curricular las bases de la intervención educativa, surgen cuestiones tales como la ya insinuada, acerca de la relación entre dicho sistema y los valores; y la cuestión afín acerca del carácter informativo, de estructuras en el ámbito del conocimiento, carácter en que todos los aspectos educativos se juzgan presentes y representados con la debida adecuación.

Por el momento cabe decir, a propósito de la cuestión segunda, que sin duda cuanto llega al educando por la intervención educativa desde el sistema curricular, puede considerarse información: se le manifiesta con rasgos o notas de contenido, y también vehiculares, susceptibles unas y otras de captarse en estructura de significados coberentes. Incluso realidades en las que los aspectos teóricos del sistema cobran determinación concreta y efectiva, como locales, mobiliario, y desde luego personas, aparecen ante el educando penetradas de significación que él capta y a su vez organiza. Más aún, los citados aspectos teóricos únicamente significan a través de su expresión efectiva y concreta, a nivel de realidad.

La relación entre el sistema curricular así concebido y los valores es cierta, y le confiere carácter general de propuesta pedagógica, a saber, hace de él patrón - pauta de comportamiento según sus propias características- por referencia a la cual es posible investigar sobre el proceso de realizarse el hombre, desde sí mismo, gracias a influjos mediadores intencionales debidos a intervención ajena; y en la raíz de esta función pedagógica hace de él, además, patrón educativo, por permitirle ser expresión afectiva y concreta (sin 
que ello implique necesaria adecuación en la propuesta) de cómo lograr dicha realización humana a través de la correspondiente intervención. Ambos niveles, o sea, los planos pedagógicos y educativo, de la propuesta general que el sistema curricular como sistema de información expresa por su estructura, remitente a la relación fundamental entre el hombre y los valores para tener sentido y con él fuerza vinculante. Es claro que si la técnica sistematiza informaciones referidas al saber hacer, debe fundarse en alguna teoría no menos sistemática, desde la cual se ponga de manifiesto cómo la acción constituye respuesta según posibilidades acordes con lo requerido y propuesto para algún fin, y éste se justifique por su valor; la técnica adquiere sentido por el conocimiento sistemático, y en él descubre el tecnólogo determinados aspectos de realidad propuesta como fin, merecedores de esti$\mathrm{ma}$, que por ello le inducen a la acción y le guían al llevarla a efecto. "Partir de una teoría y no de otra, de unos datos y no de otros, y aún formular unas determinadas hipótesis en un campo concreto frente a otras; en todo esto se ha efectuado una valoración y una elección por parte del tecnólogo". (Escámez, 1986, p. 35).

Observa Feuerstein (1979, pp. 2-6) que en las mediciones psicométricas prevalece de forma abusiva la consideración estática, según criterios de predicción basados en hipótesis de permanencia. Tales criterios han de ser sustituidos por otros que den primacía al estudio de cambios estructurales en el conocimiento $(1980$, p. 9) susceptibles de lograrse por medio de la intervención educativa, los cuales constituyan en los individuos estudiados el deseable crecimiento humano, de carácter cualitativo (1979, p. 27-29). Desde el punto de vista del sistema curricular en el que se inscribe esta intervención, la imagen del educando sólo podrá ser la de sujeto con capacidad activa para dar progresivamente estructura, cada vez más adecuada, a su asimilación de experiencias. Según Feuerstein el hecho de estructurar así las experiencias al asimilarlas, presupone mediaciones culturales que son recurso básico y decisivo de educación (1980, pp. 17-20). Al mismo tiempo, la referencia a los valores en el sistema dentro del cual se ejerce la intervención educativa, implica manifestación de que el hombre en su cultura -por la que generaliza y comparte experiencias- y en los dinamismos personales e interhumanos de su educación, está abierto y se proyecta más allá de lo ya presente y establecido: según expresa Pratt (1978, p. 137), "podría sugerirse que la esencia de la humanidad del hombre consiste en que él a través de sus acciones no sólo busca algún fin inmediato, sino también un fin trascendental, a saber, el sentido de su vida".

Resultados y procesos constituidos del orden estructural

Para comprender el psiquismo según su dimensión de conocimiento 
sobre la base de las correspondientes mediciones, es indispensable establecer diferenciación entre procesos estructurales y productos de eficiencia cognoscitiva. (Feuerstein, 1979, pp. 31 s.). Según el autor (p. 30), el concepto de eficiencia mental debe considerarse integrador en la medición de las distintas funciones cognoscitivas, y lo que recogiendo su amplitud se mide es altamente inestable. Los resultados o productos que de hecho se miden, pueden originarse menos en caracteres de capacidad cognoscitiva que reflejar otros aspectos de eficiencia mental, debidos tal vez a factores estructurales en los que el contexto participa de manera dominante y que son susceptibles de transformación por cambio del contexto, gracias a la procedente intervención educativa.

El orden curricular presupone dicho contexto, y necesita articularse en él para tomar sentido y así ofrecer a la intervención cauces de mensaje por donde ésta logre resultados optimizadores en la realización del proceso que el propio mensaje educativo intenta desarrollar. La cultura ha de ser asumida y a la vez superarse críticamente en el orden que de manera progresivista se construye, desde el orden ya construido como sistema curricular: por obra de la intervención educativa, y a nivel más profundo por autosuperación del educando como sistema. (Cfr. Sanvisens, 1983, p. 173). La superación es posible en el educando, tan sólo si algún sistema contextual le permite, por medio de informaciones, alcanzar contenidos y también hasta cierto punto dar estructura al conocimiento por despliegue de la capacidad ya presente en el modo humano de ser (y de poder constituirse y necesitarlo). Lo que por obra del educando, sistema sujeto de educación, debe lograrse con la intervención educativa según el sistema curricular en relación los sistemas de cultura contextuales asumidos, son estructuras de conocimiento que le cubran cómo iluminar desde sí mismo con experiencias las posibilidades de ser cada vez más, por referencia a los valores, lo que ya se es en la propia $y$ concreta indole bumana. Dar así sentido, curso y realización efectiva al proceso de educación, supone por una parte ejercer y por otra ser destinatario de la intervención educativa como de influjo mediador entre la cultura y la asimilación activa de experiencias; y de una y otra parte requiere cierta ardua armonía con las estructuras culturales, que las interiorice, las relacione con los fenómenos captados por experiencia, y a la vez las relativice en diálogo y confrontación entre ellas y los valores, al ir éstos mostrándose en el mismo proceso.

No parecería bien fundado interpretar de forma distinta la mediación entre la cultura y el educando, según le corresponde ejercerla al sistema curricular al traducirse en intervención educativa. El educando tiene, pues, en verdad carácter de sujeto: sus propias estructras conceptuales van haciéndose mediadoras entre las experiencias y la cultura, según los valores, por educarse con su ayuda, superándola y siendo quien, al necesitarla, además de 
imponerle caracteristicas, le hace posible desde la propia capacidad que le define y constituye. Cualquier objetivo de educación que se proponga desde el sistema curricular, debe inscribirse en el proceso cuyos fines se resumen en el de educar así al hombre. Como Wise observa, "los fines y los objetivos no son antitéticos, sino complementarios. Los objetivos visualizan y ayudan a explicar el fin, que por su parte relaciona y organiza un determinado número de objetivos" (1976, p. 280). Las estructuras culturales y subjetivas del conocimiento deben aunarse en este común proceso de educación.

La intervención educativa, seleccionadora de influjos

$\mathrm{Al}$ considerar la interdependencia de la cultura y los individuos humanos, recien sugerida, surge la cuestión de cómo formar al hombre y educarle: en el primer caso - de la formación- por el diálogo de él mismo con la cultura, y en el segundo - cuando se trata de educación- por propuesta de posibilidades en las que se determinan el contenido y la forma concreta de ese diálogo y sus resultados previsibles. Hay en los dos casos realización del hombre desde sí mismo según los valores, o sea, según lo que se muestra merecedor de estima, aunque en el segundo caso se establezcan desde el contexto curricular condiciones, a manera de cauces de realización, y se les atribuya normatividad sobre el comportamiento formativo. La cuestión se explicita como pregunta por las posibilidades condiciones y realización efectiva de un proceso optimizador en el que dos factores complementarios entre sí, a saber, el hombre educable y su contexto de cultura, actúan y se modifican o transforman reciprocamente, y al hacerlo dan curso y efectividad al citado proceso: ¿de dónde esa índole complementaria, y en ella su sentido optimizador?

Un principio de respuesta viene dado por el carácter selectivo de los influjos mediadores entre la cultura y el hombre educable, encauzados dentro de la intervención educativa por el sistema curricular, desde el mismo sistema: hay algún criterio de selección, según el cual se toman de la cultura aspectos educativos definibles por sus posibilidades y valores. Pero queda todavía por aclarar cuáles sean las raíces de tal criterio $\multimap$ de tales criterios selectivosy por tanto de su atribuida pero no menos hipotética justificación. Sobre esto no parece discutible el dato que nos muestra al hombre, educador y educable, capaz de confrontación entre la realidad efectiva y los valores en su poder de intervención educativa y en su aptitud, aún más fundamentalmente educativa, para asumir la intervención y con su ayuda realizarse humanamente. Los valores establecidos por la cultura y en algún sentido inamovibles del sístema curricular, no son marco de referencia decisivo y últi- 
mo: pueden criticarse, ya que nunca agotan la apertura humana al horizonte de lo merecedor de estima. Desde todo sistema -cultural, curricular o de cualquier índole - el sistema incluido inalienable que es cada núcleo humano de reflexión e iniciativa puede poner en tela de juicio las condiciones según las cuales se le exige relacionarse con la realidad y se le inculcan criterios interpretativos penetrados por juicios de valor. Hace falta sólo quitar la ceniza de encima de las brasas, o sea, dejar que desde la realidad misma llegue el soplo vivo de la información, para que se produzca y crezca y quizá alcance poder irresistible la llama de esta reflexión crítica y de la consiguiente superación humana. Mas ¡cómo "quitar la ceniza", hacer disponible una real y bumanizadora información, a través del sistema curricular y de la intervención educativa?

Sobre el tema, lo primero por lo que el pedagogo debe preguntarse es por el tipo de presencia cultural que en el sistema concreto de educación subyace y hasta cierto punto se explicita. Según advierte Westbury (1980, p. 90), se trata de estas preguntas, "fundamentales en la investigación sobre el curriculum: ¿Cómo se organiza y ejecuta la tarea de conocimiento y transmisión de los valores? ¿Cómo y por qué es elegido el conocimiento que la escuela juzga digno de enseñarse?" El hecho de interrogar así exige distanciamiento metódico, reserva ante formas de educación asumidas con carácter de respuesta hipotética y superable, en el camino del quehacer educativo institucional por el que es necesario opten y se comprometan, en propósito concreto y organizado, quienes educan y quienes investigan sobre cómo se educa y se debe educar. La identificación plena impediría responder bien a lo imprevisible de las situaciones (Longstreet, 1971, p. 269); y lo que es más grave, impediría desarrollar debidamente el proceso optimizador institucional a través de la reflexión crítica. Pero intentemos ver con más precisión cómo se producen los citados influjos selectivos, para acercarnos a la respuesta que piden las relaciones problemáticas entre la cultura y el educando como sistemas, tendidas a través del sistema curricular y la intervención educativa.

Nos encontramos con la referencia a criterios o principios desde los cuales cobra sentido la acción, excluyentes de las aternativas prácticas que no los asumen: "Hay en toda práctica docente urgencia por sentar principios comprensivos de la acción, que limitarán severamente el espacio de las opciones posibles". (Bolster, 1983, p. 300). A nivel de la aptitud para aprender, las enseñanzas presuponen selección adaptativa por la que se configure y concrete el sistema curricular: "En principio, cualquier estudiante puede aprender cualquier disciplina de modo significativo. Sin embargo [...] tenemos que organizar el curriculum asegurando la disponibilidad de todas las habilidades motoras necesarias y de las abstracciones primarias o secundarias que se necesiten en cualquier estadio de la secuencia del aprendizaje". (Novak, 
1982, p. 133; cfr. pp. 121, 123). Desde el punto de vista del sistema pedagógico general, la selección significa aplicar un determinado patrón educativo (y al aplicarlo surge la pregunta por su hipotética adecuación a la afinidad entre el educando y los valores): "El proceso educativo condiciona, controla y posibilita los procesos madurativos y evolutivos, [...] sobre todo, porque al ser una intervención selecionadora respecto a un patrón, dirige, orienta, selecciona y concreta los aspectos prioritarios del hombre que hay que potenciar". (Castillejo, 1986, p. 12; cfr. p. 11).

El educando se educa a si mismo, como sistema personal que recibe y procesa información: organiza conocimientos según la propia capacidad y exigencia constitutivas de construirse como hombre, y se construye guiado por la progresiva manifestación de valores y posibilidades que en dicho conocimiento se implica. Así, la comunicación de ellos - desde la cultura, a través del sistema curricular, por obra de la intervención educativa- confiere el sistema efector, es decir, al sujeto que se educa, un desarrollo de su capacidad como sistema dinámico". (Sanvisens, 1983, pp. 172 s). Tal justificación revelan los influjos que con carácter selectivo delimitan y estructuran la información transmitida al educando: se trata de establecer condiciones que le permitan desarrollarse en sentido optimizador desde sí mismo. Mas ¿qué criterios presupone el sentido optimizador buscado? Y por otra parte, ¿cómo dar estructura a las condiciones, en armonía y conexión eficaz con las posibilidades y exigencias del sujeto de educación, definible en términos de sistema personal educativos?

Contexto cultural e interacción, en el origen de estructuras conceptuales

La doble respuesta, que si resolviera las preguntas últimas también sería clave de solución para las anteriores, parece deba fundarse en lo que ya quedó sugerido: la capacidad y exigencia constitutivas del hombre, respecto de un más cualitativo siempre ulterior a lo ya asumido por él en la propia realidad efectiva de sus informaciones, criterios y constitución personal; y a la vez, su necesidad ineludible de estructurar informaciones, recibidas a partir de experiencias - y con ello guiarse en la realización de sí mismogracias a la mediación ofrecida por esquemas coherentes de cultura desde el interior de relaciones interbumanas, en las que tales esquemas se recogen y someten a confrontación crítica.

Hablar aquí de "esquemas", como también de "estructuras", es señalar alguna relación $u$ orden relacional que subordinan aspectos, elementos o partes a cierta unidad común; en el caso de los esquemas, por referencia al contenido que recibe significación dentro de la unidad; y en caso de las estructuras, por significarse la interdependencia de relaciones, constitutiva 
del orden y según él de la unidad como sistema. La cultura sintetiza interpretaciones, y es su propia síntesis compartida por los miembros de un mismo grupo, individuos humanos adscritos, en su forma de conocer, al cauce cultural común que tales interpretaciones establecen. Por la intervención educativa, además de transmitirse informaciónes según esquemas culturales, es preciso dar ocasión y medios para la reflexión crítica, a través de la cual se los enjuicie, y según el modelo de valores que la cultura nunca logra agotar, se los transforme en estructuras de conocimiento, individuales y colectivas, progresivamente capaces de guiar al hombre en la tarea de construirse como hombre bumano.

Un posible bosquejo de explicitación referida al propósito de formar así las estructuras de conocimiento, viene dado por las conclusiones que expone Feuerstein (1980, pp. 26-35), para quien los estímulos, asumidos a través de mediaciones en el aprendizaje estructural, es preciso desarrollen en sentido humano y humanizador el conocimiento selectivo, la construcción anticipadora de realidad, el comportamiento imitativo y la novedad en la repetición; todo lo cual implica progresiva adaptación a la temporalidad y una cada vez mayor eficiencia comparativa. Pero bajo los distintos aspectos de este desarrollo lo que se busca, a través del aprendizaje por experiencias sometidas a mediación, ha de ser la formación de la autonomía (o.c., p. 70); $\mathrm{y}$ sin duda, todo lo dicho presupone trascendencia intencional -superación de la realidad efectiva por el conocimiento y la decisión eficaz- en la relación educativa, mediadora de experiencias. (Cfr. pp. 20-22).

La mediación educativa apuntada sugiere reconocer, con Novak (1982, p. 147), la insustituible función del "aprendizaje receptivo significativo a través de secuencias de isntrucciones que estén planificadas con cuidado". Pero la primacía pedagógica del educando como sujeto del quehacer educativo obliga a ver en el "modelo del aprendizaje problemático, por medio del descubrimiento", un más hondo significado teórico; y a la vez es justo señalar que en el citado modelo "la interrelación entre teoría y práctica es perfectamente factible, más aún, la teoría comprende - comprehende-la práctica, la implica en sí misma”. (Vázquez, 1982, p. 504). A nivel de práctica, la teoría impone reflexión por la que el mismo sujeto del desarrollo personal descubra sus posibilidades, condiciones y exigencias en sentido optimizador, y así se construya humanamente. "La educación sistémica no sólo ha de hacer posible que cuanto antes el educando sea el artífice principal de su propio progreso educativo, sino que en los procesos comunicativos interpersonales los mensajes deberán provocar el 'diálogo interno', la reflexión personal que convierta en activa la participación...” (Sarramona, 1983, p. 54).

Las estructuras de conocimiento no pueden alcanzar de manera progresiva la adecuación que su índole humana y humanizadora requiere, sino 
por la mediación educativa que debidamente informa y a la vez suscita y favorece con eficacia la reflexión. "Lo que se experimenta en la familia, la escuela, la sociedad, y viene expresado por automatismos de comportamiento, debe hacerse visible y ser tema de reflexión en el contexto de situaciones y problemas actuales. (Garlicha, 1985, p 369; cfr. p. 378). En la intervención educativa, los influjos mediadores orientados a la formación de estructuras de conocimiento revelan sentido, y cobran eficacia, al suscitar experiencias por las que el propio sujeto del proceso capta de modo presencial, concreto y coherente la realidad y el valor. "Cada uno tiene sin cesar experiencias. Lo experimentado se reconoce, se sitúa dentro de conjuntos en orden jerárquico, se clasifica. De cómo se realicen tales hechos depende, en definitiva, el horizonte de lo que es experimentado". (Thiemann, 1983, p. 204). Por eso la formación de estructuras que permitan asumir experiencias de modo bumano y humanizador, debe lograrse por mediación educativa sobre las mismas experiencias, con propósito doble y unitario: mostrar el trasfondo valorativo que les da significación, y establecer en consecuencia condiciones efectivas para la progresiva adecuación estructural entre el conocimiento y el valor, promovida a nivel del orden según el cual el educando organiza dichas experiencias.

Orden y experiencias interhumanas: sentido pedagógico de su dialéctica

Hablar sobre la formación del orden estructural en el conocimiento implica tener que plantearse con rigor la pregunta por los valores educativos. Quienes emiten por medio de la intervención mensajes que expresan al receptor la propuesta de cómo por el conocimiento deba construirse en sentido humano optimizador, y procuran condicionarle según ella, han de participar y admitirle en la confrontación entre el orden constitutivo del hombre humano tal como dichos mensajes lo interpretan en relación con la cultura -y tal como intentan hacerlo operativo - y ese más cualitativo ulterior al que siempre el hombre está abierto. La reflexión critica, al establecer con validez y eficacia dicha confrontación y desarrollarla, hace imposible en igual medida que la manipulación prevalezca para los educandos, y también para los educadores allí donde la sociedad les sustituye como responsables de los propios criterios, se remueve entonces el obstáculo que impedía reconocer los valores según su índole estimable y tomar las decisiones correspondientes: aquel a quien la manipulación engaña, si se le hiciese comprender cómo y por qué le han llevado a tergiversar o confundir su deliberación, justamente en ese momento dejaría de decidir como había decidido". (Touriñan, 1984, pp. 94 s.).

Por de pronto requiere examen el contexto inmediato de la intervención 
educativa que es el sistema curricular, en sus dimensiones manifiesta y oculta; hace falta ver de dónde recibe la intervención su significado, para comprenderlo y realizar la correspondiente valoración crítica. Si lo manifiesto pide examen profundizador, los aspectos ocultos, con su vaguedad, su inmediatez o cercania dondequiera se produzcan decisiones, su carácter envolvente, su consistencia y penetración y su normatividad a partir de valores definidos por la cultura (cfr. Vázquez, 1938, p. 173), piden atención particular, y respuesta coherente y eficaz que debe traducirse en "tres funciones, a saber, ampliación y liberación de la consciencia, promoción de una actitud crítica e impulso de la intencionalidad respecto de hábitos, reglas y valores que orienten el comportamiento del educando" (p. 176).

Por la reflexión crítica han de cobrar evidencia interrogativa y confrontarse las claves de significación que presiden el sistema curricular desde la cultura; porque "los elementos de mayor importancia dentro de toda situación social son las significaciones compartidas, que los participantes toman de su proceso de interacción y que en definitiva les configuran el comportamiento". (Bolster, 1983, p. 303). Desde el punto de vista de Eco, "la historia, con su vitalidad voraz, vacia y llena las formas, las priva y dota de significado" (1972, p. 352). ¿Cómo descubrir por confrontación crítica del lenguaje sentido humano y humanizador - esencial y eficiente, en la intervención educativa desde el sistema contextual- que guíe la formación de estructuras de conocimiento? La razón sólo funcional, sin ámbito de luz que la abra a preguntas y respuestas ulteriores cada vez más profundas, no basta de ningún modo: según Heidegger, "es la más porfiada enemiga del pensar" (1969, p. 221). Si el hombre se define por su apertura y proyección más allá de lo conocido y las formas de conocerlo, debe dirigir su mirada bacia donde la superación misma aparece y al aparecer ilumina los significados (Cfr. o. c. p. 307). Dando cabida a la comunicación e iniciativa humana en esta búsqueda, resultan esclarecedoras la interpretación y la subyacente propuesta y actitud sugeridas por el propio Heidegger: "El lenguaje habla. El hombre habla en la medida en que se ajusta al lenguaje. El ajustarse es escuchar. Se escucha en la medida en que se obedece al mandato del silencio" (1960, pp. 32 s.). 


\section{BIBLIOGRAFIA}

BOLSTER, A.S., Jr. (1983). Toward a More Effective Model of Research on Teaching. En Harvard Educational Review (vol. 53) pp. 294-308.

CASTILLEJO BRULL, J.L. (1986). Proceso educativo y construcción humana. En Conceptos y propuestas (III), pp. 2-28. Valencia: Papers d'Educació/Nau Llibres.

ECO, U. (1972). La estructura ausente. Introducción a la semiótica. Barcelona: Lumen.

ESCAMEZ, J. (1986). Los valores en la Pedagogía de la Intervención. En Conceptos y propuestas (III), pp. 29-48. Valencia: Papers d'Educació / Nau Llibres.

FEUERSTEIN, R., et al. (1979). The Dynamic Assessment for Retarded Performers. The Learning Potential Assessment Device Theory, Instruments and Technique. Glenview, III.: Scott, Foresman and Co.

FEUERSTEIN, R., et al, (1980). Instrumental Enricbment. An Intervention Program for cognitive Modifiability. Glenview, III.: Scott, Foresman and Co.

GARLICHS, A. (1985). Selbsterfahrung als Bildungsaufgabe der Schule. En Zeit schrift für Pädagogik (vol. 31) pp. 365-383.

HEIDEGGER, M. (1960). Unterwegs zur Sprache. Pfullingen: Neske.

HEIDEGGER, M. (1969). Sendas Perdidas. Buenos Aires: Losada.

LONGSTREET, W.S. (1971). Toward a Curriculum Incorporating Process. En Educational Theory (vol. 21 n. 3) pp. 261-273.

NOVAK, J.D. (1982). Teoría y práctica de la educación. Madrid: Alianza.

PRATT, D. (1978). System Theory, Systems Technology, and Curriculum. En Journal of Educational Thought (vol. 12) pp. 131-152.

SANVISENS, A. (1983). Concepción sistémico-cibernética de la educación. En Teoría de la educación I. (El problema de la educación), pp. 161-186. Murcia: Límites.

SARRAMONA, J. (1983). La educación como sistema de comunicación. En Teoría de la educación I. (El problema de la educación), pp. 43-62. Murcia: Límites.

SCHWAB, J.J. (1971). The Practical: A Language for Curriculum. En Levit, M. (Ed.), Curriculum, pp. 306-330. Chicago, Urbana: University of Illinois Press.

THIEMANN, F. (1983). Zur Funktion alltäglicher Interpretationsarbeit: Wie Schulerfahrung organisiert wird. En Bildung und Erziehung (vol. 36) pp. 203-212.

TOURIÑAN LOPEZ, J.N. (1984). Delimitación de la intervención educativa como acción. En Revista Española de Pedagogía, vol. 42 (n. 163) pp. 79-97.

VAZQUEZ GOMEZ, G. (1982). El principio curricular de la relación entre la teoría y la práctica. Aplicación a la formación de Profesores y de Pedagogos. En Bordón, vol. 34 (n. 245) pp. 495-516.

VAZQUEZ GOMEZ, G. (1985). Currículum oculto y manifiesto. En Condicionamientos socio-políticos de la educación, pp. 167-187.

WISE, R.I. (1976). The Use ob Objectives in Curriculum Planning. A Critique of Planning by Objectives, En Curriculum Theory Network (vol. S, n. ${ }^{\circ}$ pp. 280-289.

WESTBURY, I. (1980). Schooling as an Agency of Education: Some Implications for Curriculum Theory. En Dockrell, W.B., Hamilton, D. (Eds.), Rethinking Educational Research, pp. 88-114. London: Hodder and Stoughton. 